\title{
Patterns of Articular Cartilage Thickness in Pediatric and Adolescent Knees: A Magnetic Resonance Imaging-Based Study
}

\author{
Sreetha Sidharthan, M.D., Annie Yau, B.A., Bryan Aristega Almeida, B.A., \\ Kevin G. Shea, M.D., Harry G. Greditzer IV, M.D., Kristofer J. Jones, M.D., and \\ Peter D. Fabricant, M.D., M.P.H.
}

\begin{abstract}
Purpose: To establish normative values for articular cartilage thickness in pediatric and adolescent knees using magnetic resonance imaging (MRI) and investigate for any associations with age and skeletal maturity. Methods: MRI scans were analyzed in patients 7 to 18 years old without osteochondral lesions, chondral wear/pathology, intra-articular fractures, or history of knee surgery. Measurements of articular cartilage thickness at the patella (medial facet, lateral facet, median ridge), femur (medial condyle, lateral condyle, lateral trochlea), and tibia (medial plateau, lateral plateau) were made on axial, coronal, and sagittal MRI. Descriptive statistics were used to calculate mean cartilage thickness by age and sex. Analysis of variance with repeated measures, analysis of covariance, independent samples $t$ test, and linear regression were performed to determine differences in mean cartilage thickness by anatomic location, sex, physeal status, and age, respectively. Results: A total of 240 knee MRI scans were included. Articular cartilage was thickest at the patella and did not vary with age or skeletal maturity. On the femur, articular cartilage was thickest at the lateral trochlea with mean cartilage thickness of $4.4 \pm 1.4 \mathrm{~mm}$ in male patients and $3.6 \pm 1.3 \mathrm{~mm}$ in female patients $(P<.001)$. Patients with open distal femoral physes had significantly thicker cartilage at the medial femoral condyle, lateral femoral condyle, and lateral trochlea compared to patients with closing/closed physes $(\mathrm{P}<.001)$. Linear regression analysis revealed a significant inverse association between cartilage thickness at the femur and age. Conclusions: In pediatric and adolescent knees, articular cartilage is thickest at the patella, where it does not strongly correlate with age. In contrast, there is a strong inverse association between increasing age and articular cartilage thickness of the distal femoral condyles. Clinical Relevance: The longitudinal reference data presented in this study can aid in pre-operative interpretation of knee cartilage under pathologic conditions in pediatric and adolescent patients.
\end{abstract}

$\mathbf{F}$ ocal articular cartilage lesions of the knee are challenging problems faced by sports medicine and pediatric orthopaedic surgeons alike. In children and

From the Divisions of Pediatric Orthopaedic Surgery (S.S., A.Y., B.A.A., P.D.F.) and Radiology $\theta$ Imaging (H.G.G.), Hospital for Special Surgery, New York, New York; David Geffen School of Medicine at UCLA, Los Angeles (K.J.J.); and Stanford University School of Medicine, Stanford (K.G.S.), California, U.S.A.

The authors report the following potential conflicts of interest or sources of funding: K.G.S. reports other from Ossur and Vericel, outside the submitted work; and is a board/committee member of the American Academy of Orthopaedic Surgeons, Pediatric Orthopedic Society of North America, PRiSM (Pediatric Research in Sports Medicine), and ROCK (Research for Osteochondritis Dissecans of the Knee). K.J.J. reports other from Arthrex, CONMED Linvatec, JRF Ortho, Vericel, Aesculap/B. Braun, Zimmer, Musculoskeletal Transplant Foundation, outside the submitted work; and is a board/committee member of the American Orthopaedic Society for Sports Medicine. P.D.F. reports editorial or governing board member of Clinical Orthopaedics and Related Research; and board or committee member of the Pediatric Orthopaedic Society of North America, Pediatric Research in Sports adolescents, these injuries are commonly due to osteochondritis dissecans, avascular necrosis, patellofemoral dislocation, or trauma. ${ }^{1,2}$ Growing participation

Medicine Society, and ROCK. Full ICMJE author disclosure forms are available for this article online, as supplementary material.

Investigation performed at the Hospital for Special Surgery, New York, New York, U.S.A.

Presented at the 2020 Annual Meeting of the Pediatric Research in Sports Medicine (PRiSM) Society.

Received May 4, 2020; accepted September 30, 2020.

Address correspondence to Peter D. Fabricant, M.D., M.P.H., Pediatric Orthopaedic Surgery $\theta$ Sports Medicine, Hospital for Special Surgery, 535 East 70th St., New York, NY 10021.

(C) 2020 THE AUTHORS. Published by Elsevier Inc. on behalf of the Arthroscopy Association of North America. This is an open access article under the CC BY-NC-ND license (http://creativecommons.org/licenses/by-nc-nd/4.0/). 2666-061X/20663

https://doi.org/10.1016/j.asmr.2020.09.029 
in organized sports such as soccer and basketball has led to a corresponding increase in incidence of knee injuries in adolescents. ${ }^{3,4}$ Magnetic resonance imaging (MRI) studies have shown that chondral lesions are the most prevalent injuries in skeletally immature patients with knee trauma. ${ }^{5}$ Left untreated, these lesions can lead to progressive articular cartilage degeneration, early-onset osteoarthritis, and functional disability. ${ }^{6-8}$

Understanding cartilage anatomy and morphology is important to evaluating and treating osteochondral and chondral lesions. Longitudinal reference data on cartilage development can aid in interpretation of knee cartilage under pathologic conditions in children and adolescents. In addition, quantifying native cartilage thickness may help match donor and recipient sites for osteochondral autograft transplantation system (OATS $)^{9}$ and osteochondral allograft transplantation (OCA). ${ }^{2}$

The purpose of the current study was to establish normative values for articular cartilage thickness in pediatric and adolescent knees using MRI and investigate for any associations with age and skeletal maturity. ${ }^{10}$ We hypothesized that articular cartilage thickness at the knee is inversely correlated with age and skeletal maturity.

\section{Materials}

\section{Study Cohort}

This study was carried out at an urban academic tertiary care orthopaedic facility and approved by the hospital's institutional review board (number: 2014189). Picture archiving and communication system records from January 2008 to December 2018 were queried for knee MRI in patients aged 7 to 18 years old. Radiology reports were randomly reviewed until 20 patients (10 male, 10 female) from each year of age that met inclusion criteria were identified. Patients were excluded if they had osteochondral lesions, chondral wear or pathology, intra-articular fractures, history of knee surgery, inflammatory arthropathy, patellar subluxation, and/or motion degradation during imaging. Patients with acute knee injuries such as anterior cruciate ligament (ACL) or meniscal tears were included in the final analysis only if they did not have concomitant chondral injury.

\section{Measurement of Articular Cartilage Thickness}

All MRIs were performed on a standard clinical 1.5-T or 3.0-T MRI scanner with 3-mm image slices (GE Healthcare, Waukesha, WI), using a standardized quadrature or 8-channel knee coil (Invivo Inc., Gainesville, FL) and the knee in extension. Measurements of articular cartilage thickness were made on proton density-weighted, high-resolution, fast-spin-echo sequences. ${ }^{10-13}$ Using Sectra ID57 tools, thickness was measured perpendicular to the bone/cartilage interface, from the level of the subchondral bone to the superficial articular cartilage surface.

Various axial, coronal, and sagittal cuts were selected for quantification of cartilage thickness (Fig 1). An axial image through the level of thickest patellar cartilage was used. The coronal cut was determined by identifying a frontal plane bisecting the femur on the axial image, and Cahill zones 1, 2, 3, 4, and 5 were delineated. ${ }^{14}$ Three sagittal cuts were selected: the first bisected the patella at the median ridge on the axial image, the second localized to center of the medial femoral condyle on the coronal image, and the third corresponded with the center of the lateral femoral condyle on the coronal image. On the sagittal slices of the medial and lateral femoral condyles, Cahill zones A, $\mathrm{B}$, and $\mathrm{C}$ were outlined. ${ }^{14}$

Measurements of cartilage thickness at the patella were made at the midpoint of the medial facet on axial slice, midpoint of the lateral facet on axial slice, and the median ridge on sagittal MRI. Cartilage thickness at the medial femoral condyle was measured at 5 regions on coronal and sagittal images: midpoint of the medial condyle and midpoints of Cahill zones 1 (MIDl), 2 (MID2), B (MIDB), and C (MIDC). Similarly, measurements of cartilage thickness of the lateral femoral condyle were made at the midpoint of the lateral condyle and midpoints of Cahill zones 4 (MID4), 5 (MID5), B (MIDB), and C (MIDC). Measurement of the lateral trochlear cartilage was made at midpoint of Cahill zone A (MIDA). On the tibia, measurements were made at the midpoint of the medial tibial plateau and the midpoint of the lateral tibial plateau (LTP) on the coronal image.

Two senior medical students (S.S., A.Y.) recorded the cartilage thickness measurements under the direction of one attending orthopaedic surgeon (P.D.F.) and one fellowship-trained musculoskeletal radiologist (H.G.G.) who made the final measurements. For 24 MRI scans, 2 belonging to each year of age, measurements were carried out in duplicate for determination of interrater reliability. The remaining 216 scans were divided between the 2 investigators.

\section{Skeletal Maturity}

Skeletal maturity was evaluated by the status of the proximal tibial and distal femoral growth plates on coronal and sagittal T1 MRI by 2 investigators (S.S., A.Y.). ${ }^{15}$ Each physis was categorized as "open" or "closing/closed." A normal growth plate appears as a uniform low-signal intensity line extending across the width of the bone. Physes were classified as "open" if the entire growth plate could be visualized without a central closure. If the characteristic low signal of an active growth plate could not be visualized extending across the width of the proximal tibia or distal femur, 


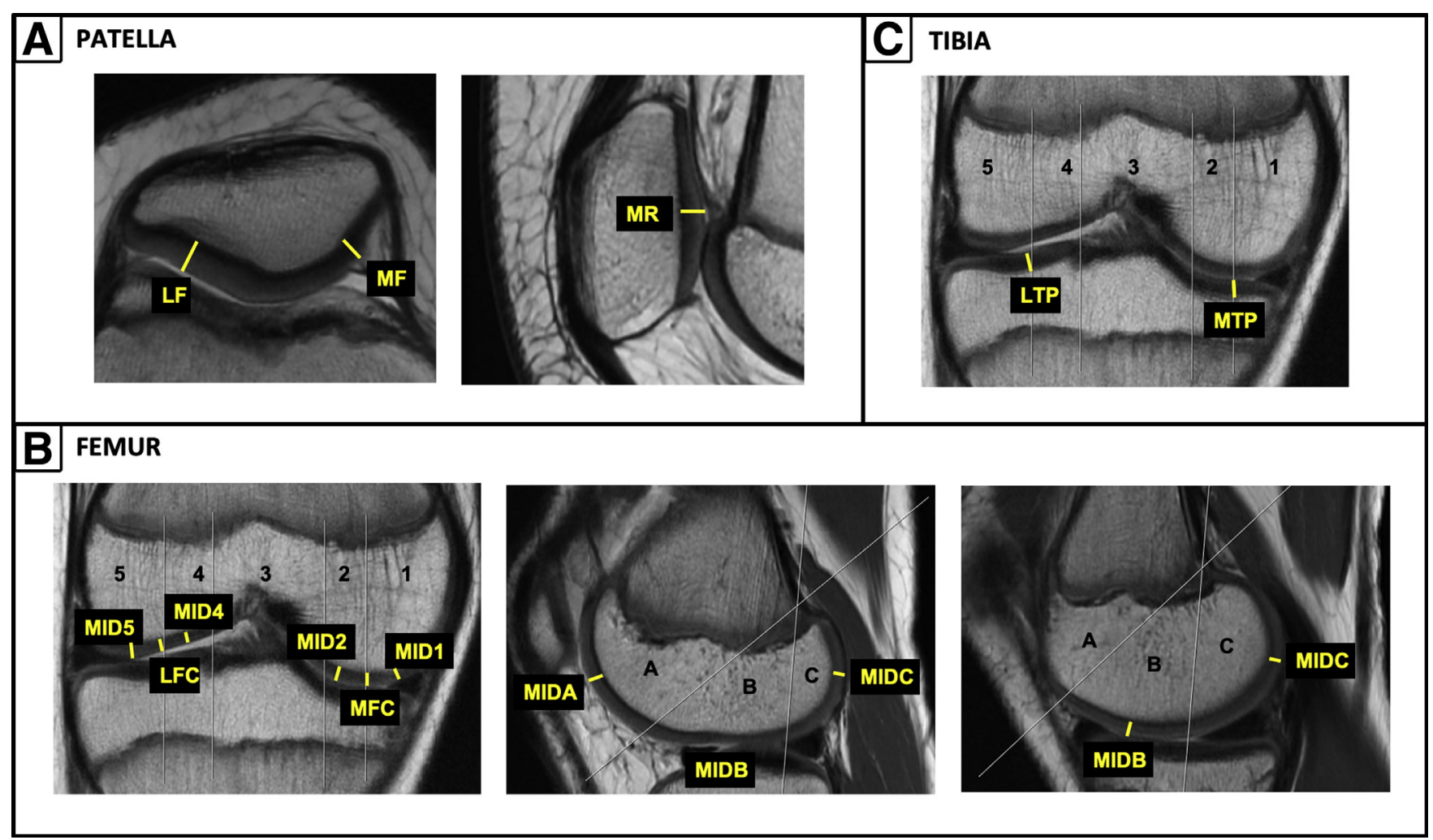

Fig 1. MRI measurements of articular cartilage thickness. Articular cartilage thickness was measured on axial, coronal, and sagittal MRI of the knee. In this figure, a left knee with an open physis was measured. (A) Measurements of cartilage thickness at the patella were made at the midpoint of the medial facet (MF), midpoint of the lateral facet (LF), and the median ridge (MR) on axial and sagittal MRI scans. (B) For the medial femoral condyle, cartilage thickness was measured at five regions: midpoint of the medial condyle (MFC) and midpoints of Cahill zones 1 (MID1), 2 (MID2), B (MIDB), and C (MIDC). Similarly, measurements of cartilage thickness of the lateral femoral condyle were made at the midpoint of the lateral condyle (LFC) and midpoints of Cahill zones 4 (MID4), 5 (MID5), B (MIDB), and C (MIDC). Cartilage thickness at the lateral trochlea was measured at midpoint of Cahill zone A (MIDA). (C) Cartilage thickness at the medial tibial plateau (MTP) and lateral tibial plateau (LTP) were made on the coronal slice. (MRI, magnetic resonance imaging.)

then it was classified as "closed/closing." For measurements of articular cartilage thickness of the patella and distal femur, skeletal maturity of knees was categorized by the status of the distal femoral growth plate. For tibial-sided measurements, knees were categorized by the status of the proximal tibial growth plate.

\section{Statistical Analysis}

All data were collected using Excel (Microsoft, Redmond, WA) and analyzed using SPSS version 22.0 (IBM Corp., Armonk, NY). Interrater reliability was calculated for each measurement using intraclass correlation coefficient (ICC) based on absolute agreement and was classified according to the criteria described by Landis and Koch. ${ }^{16}$ Measurements were carried out in duplicate on 24 MRI scans for the purpose of determining ICC scores. Descriptive statistics were used to calculate mean articular cartilage thickness by age and sex at each of the anatomic sites. One-way analysis of variance with repeated measures and post-hoc analysis using Bonferroni correction were used to determine if mean cartilage thickness varied by anatomic site in the knee. One-way analysis of covariance controlling for age and independent samples t-test were used to investigate differences in cartilage thickness by sex and skeletal maturity, respectively. Linear regression analysis was performed to evaluate the association between age and cartilage thickness, and hierarchical linear regression was used to introduce gender into this model.

All analyses were 2-tailed and used $P=.05$ as the threshold for statistical significance. As this study was designed to be primarily descriptive, an a priori power calculation was not performed. ${ }^{17}$

\section{Results}

A total of 240 MRIs (120 male, 120 female) were evaluated and analyzed (Table 1). Mean age was $13.0 \pm$ 3.4 years. The most common MRI diagnosis among the included patients was ACL injury $(17.5 \%, 42 / 240)$, and $16.3 \%(39 / 240)$ of MRIs had negative findings 
Table 1. MRI Diagnoses of Included Subjects

\begin{tabular}{lr}
\hline \multicolumn{1}{c}{ Primary MRI Finding } & $\mathrm{N}(\%)$ \\
\hline ACL hyperintensity/sprain/tear & $42(17.5 \%)$ \\
Normal & $39(16.3 \%)$ \\
Bone marrow edema & $32(13.3 \%)$ \\
Meniscal tear, scarring of & $23(9.6 \%)$ \\
$\quad$ meniscocapsular junction & \\
Infrapatellar/medial parapatellar plica, & $18(7.5 \%)$ \\
$\quad$ thickened/scarred & \\
Fat pad edema/impingement & $17(7.1 \%)$ \\
MCL sprain/scarring/tear & $11(4.6 \%)$ \\
Soft-tissue edema & $11(4.6 \%)$ \\
Patellar tendinosis & $10(4.2 \%)$ \\
Discoid meniscus, hyperintensity/tear & $8(3.3 \%)$ \\
IT band edema/scarring & $7(2.9 \%)$ \\
Sinding-Larsen-Johansson, Osgood-Schlatter & $7(2.9 \%)$ \\
Ganglion cyst & $4(1.7 \%)$ \\
Metaphyseal non-ossifying fibroma & $3(1.3 \%)$ \\
Popliteal cyst, ruptured & $3(1.3 \%)$ \\
Infrapatellar/prepatellar bursitis & $2(0.8 \%)$ \\
Pes anserine thickening/injury & $2(0.8 \%)$ \\
LCL injury & $1(0.4 \%)$ \\
\hline
\end{tabular}

NOTE. No subject had any associated chondral or osteochondral pathology.

ACL, anterior cruciate ligament; IT, iliotibial band; LCL, lateral collateral ligament; MCL, medial collateral ligament.

(Table 1). No subject had any associated chondral or osteochondral pathology. ICC scores ranged from 0.66 to 0.94 indicating substantial to almost perfect interrater reliability for measuring cartilage thickness at the various anatomic locations (Table 2).

Taken together as a cohort mean, articular cartilage was thickest at the patella, followed by the lateral trochlea, the medial and lateral femoral condyles, and the lateral and medial tibial plateaus (Fig 2). The medial and lateral facets of the patella exhibited the thickest knee cartilage measuring $5.1 \pm 1.2 \mathrm{~mm}$ and $5.0 \pm 1.0 \mathrm{~mm}$, respectively. On the femur, cartilage thickness was greatest at the lateral trochlea measuring an average of $4.0 \mathrm{~mm} \pm 1.3 \mathrm{~mm}$. A repeated-measures analysis of variance with a Greenhouse-Geisser correction determined that mean cartilage thickness differed statistically significantly between the various anatomic locations $(P<$ $.001)$. Post hoc tests using the Bonferroni correction revealed statistically significant differences in all pairwise comparisons $(P \leq .01)$ with the exception of 2 . There was no significant difference in cartilage thickness between the medial and lateral facets of the patella $(5.1 \pm 1.2 \mathrm{~mm}$ vs $5.0 \pm 1.0 \mathrm{~mm}, P=1.0)$ or between the lateral femoral condyle and lateral tibial plateau $(3.0 \pm 0.7 \mathrm{~mm}$ vs $3.1 \pm 0.8 \mathrm{~mm}, P=1.0)$.

Overall, male patients had thicker articular cartilage than female patients at the patella, femur, and tibia, and this finding was found to be statistically significant by analysis of covariance controlling for age (Table 3).
There was no difference in cartilage thickness at the medial facet and median ridge of the patella between patients with open versus closed/closing distal femoral physes (Table 3). At the lateral patellar facet, knees with open physes had on average thinner cartilage compared with knees with closed/closing distal femoral physes by $0.5 \mathrm{~mm}(P=.01)$. In comparison, knees with open distal femoral physes demonstrated significantly thicker cartilage at the lateral trochlea by an average of 1.6 $\mathrm{mm}$, at the medial femoral condyle by $1.2 \mathrm{~mm}$, and at the lateral femoral condyle by $0.9 \mathrm{~mm}$ (Table 3). This was similarly true at the tibia: articular cartilage at the medial and lateral tibial plateaus was thicker in patients with open physes than closing/closed proximal tibial physes but only by an average of $0.6 \mathrm{~mm}$ and $0.4 \mathrm{~mm}$, respectively.

Articular cartilage thickness at the patella was not strongly associated with age (Fig 3, Table 4). By linear regression, age was not a significant predictor of cartilage thickness at the lateral facet and median ridge. Although the linear regression model correlating age and medial facet thickness was found to be statistically significant $(\mathrm{y}=-0.05 \mathrm{x}+5.7, P=.04)$, this association was likely driven by thicker cartilage among the younger years of age. Furthermore, age only accounted for $1 \%$ of the variation in medial facet cartilage thickness.

In contrast, articular cartilage thickness was inversely correlated with age at the femur (Fig 4, Table 4). In linear regression analysis, age explained $62 \%$ of the variance in cartilage thickness at the lateral trochlea $(\mathrm{y}=-0.31 \mathrm{x}+8.0, P<.001), 61 \%$ of the variance at the medial femoral condyle $(\mathrm{y}=-0.24 \mathrm{x}+6.5, P<$ $.001)$, and $63 \%$ of the variance at the lateral femoral

Table 2. Interrater Reliability of Articular Cartilage Thickness Measurements

\begin{tabular}{lc}
\hline \multicolumn{1}{c}{ Anatomic Location } & $\begin{array}{c}\text { ICC }(2,1) \text { Score } \\
(95 \% \mathrm{CI})\end{array}$ \\
\hline Patella & $0.86(0.60-0.94)$ \\
Medial facet (MF) & $0.87(0.61-0.95)$ \\
Lateral facet (LF) & $0.85(0.68-0.93)$ \\
Median ridge (MR) & \\
Femur & $0.80(0.60-0.91)$ \\
Lateral trochlea (MIDA) & $0.92(0.82-0.94)$ \\
Medial femoral condyle (MID1) & $0.94(0.87-0.97)$ \\
Medial femoral condyle (MID2) & $0.79(0.58-0.90)$ \\
Medial femoral condyle (MIDB) & $0.90(0.77-0.95)$ \\
Medial femoral condyle (MIDC) & $0.87(0.73-0.94)$ \\
Lateral femoral condyle (MID4) & $0.97(0.93-0.99)$ \\
Lateral femoral condyle (MID5) & $0.84(0.67-0.93)$ \\
Lateral femoral condyle (MIDB) & $0.89(0.71-0.96)$ \\
Lateral femoral condyle (MIDC) & \\
Tibia & $0.66(0.36-0.84)$ \\
Lateral tibial plateau (LTP) & $0.80(0.52-0.91)$ \\
Medial tibial plateau (MTP) & coefficient.
\end{tabular}




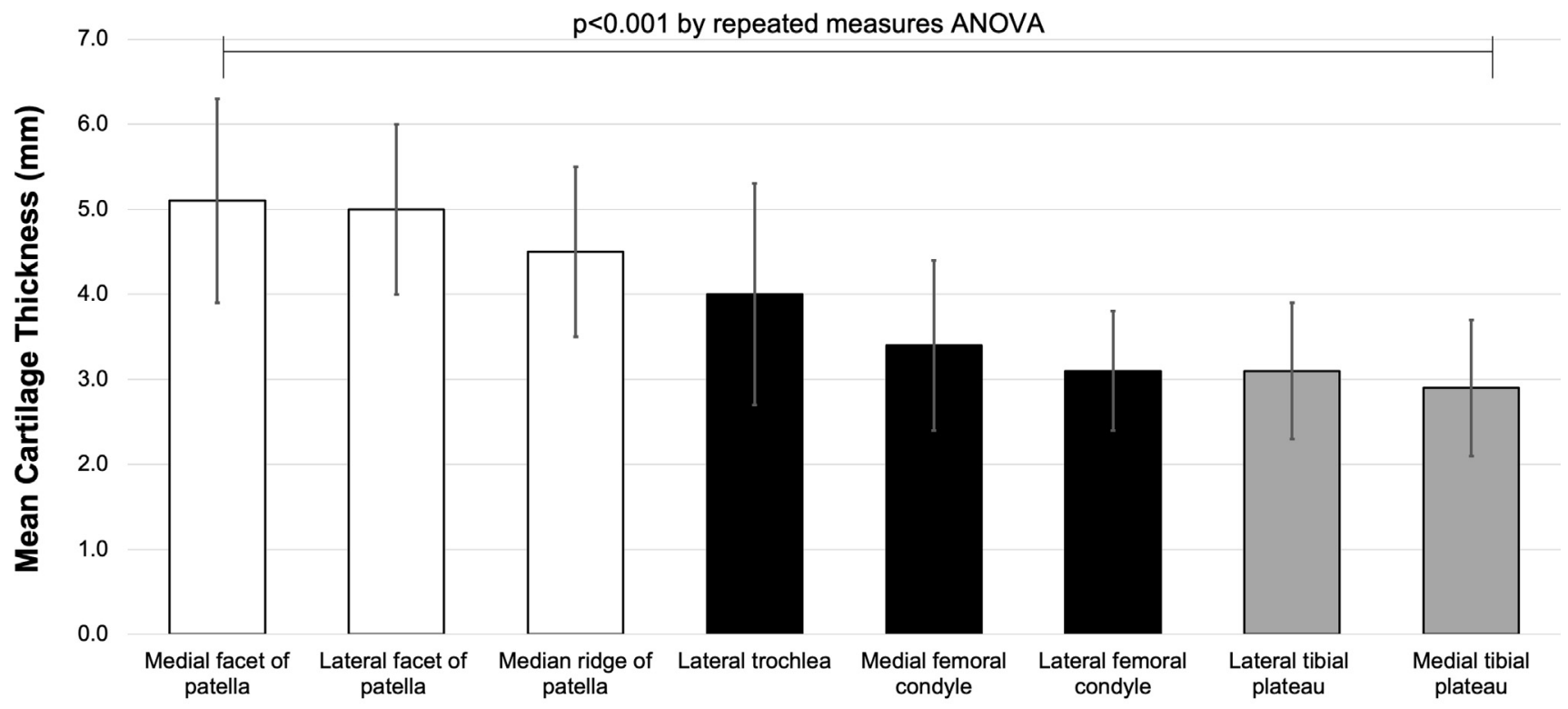

Anatomic Location

Fig 2. Mean articular cartilage thickness by anatomic location. Mean cartilage thickness at the patella, femur, and tibia are presented in a bar graph. Error bars represent standard deviation. A repeated-measures ANOVA with a Greenhouse-Geisser correction determined that mean cartilage thickness differed statistically significantly between the 8 anatomic locations $(P<$ $.001)$. Post hoc tests using the Bonferroni correction revealed statistically significant differences in all pairwise comparisons $(P \leq$ .01 ) with the exception of 2 . There was no significant difference in articular cartilage thickness between the medial and lateral facets of the patella or between the lateral femoral condyle and lateral tibial plateau. (ANOVA, analysis of variance.)

condyle $(\mathrm{y}=-0.15 \mathrm{x}+5.1, P<.001)$. When we performed hierarchical linear regression analysis, including sex as a variable into these models slightly increased the adjusted $\mathrm{R}^{2}$ value, while maintaining the impact of age (Table 4).

Cartilage thickness at the tibia was also inversely correlated with age (Fig 5, Table 4). Although this association was statistically significant, age only explained $6 \%$ of the variance in cartilage thickness at the lateral tibial plateau $(\mathrm{y}=-0.06 \mathrm{x}+3.9, P<.001)$ and $27 \%$ of the variance at the medial tibial plateau $(\mathrm{y}=-0.12 \mathrm{x}+4.4, P<.001)$. Hierarchical linear regression analysis with sex as an additional variable minimally increased the adjusted $\mathrm{R}^{2}$ value (Table 4 ).

Table 3. Mean Articular Cartilage Thickness by Sex and Skeletal Maturity

\begin{tabular}{|c|c|c|c|c|c|c|}
\hline \multirow[b]{2}{*}{ Anatomic Location } & \multicolumn{3}{|c|}{ Sex } & \multicolumn{3}{|c|}{ Physeal Status* } \\
\hline & Male $(\mathrm{n}=120)$ & Female $(\mathrm{n}=120)$ & $P$ Value $\dagger$ & Open & Closed/Closing & $P$ Value $\ddagger$ \\
\hline \multicolumn{7}{|l|}{ Patella } \\
\hline Median ridge & $4.7 \pm 1.1$ & $4.3 \pm 0.9$ & .002 & $4.4 \pm 0.9$ & $4.6 \pm 1.3$ & .3 \\
\hline \multicolumn{7}{|l|}{ Femur } \\
\hline Lateral trochlea & $4.4 \pm 1.4$ & $3.6 \pm 1.2$ & $<.001$ & $4.5 \pm 1.3$ & $2.9 \pm 0.6$ & $<.001$ \\
\hline \multicolumn{7}{|l|}{ Tibia } \\
\hline Lateral tibial plateau & $3.3 \pm 0.7$ & $3.0 \pm 0.8$ & $<.001$ & $3.3 \pm 0.7$ & $2.9 \pm 0.8$ & .001 \\
\hline Medial tibial plateau & $3.1 \pm 0.9$ & $2.7 \pm 0.5$ & $<.001$ & $3.1 \pm 0.8$ & $2.5 \pm 0.4$ & $<.001$ \\
\hline
\end{tabular}

NOTE. $P$ values in bold are statistically significant $(P \leq .05)$.

ANCOVA, analysis of covariance.

*For measurements at the patella and femur, knees were categorized by the status of the distal femoral physis as open ( $\mathrm{n}=173)$ versus closing/ closed $(n=67)$. For tibial-sided measurements, knees were categorized by the status of the proximal tibial growth plate as open $(n=156)$ versus closing/closed $(\mathrm{n}=84)$.

${ }^{\dagger} P$ values from ANCOVA controlling for age

${ }^{\ddagger} P$ values from independent samples $t$ test. 


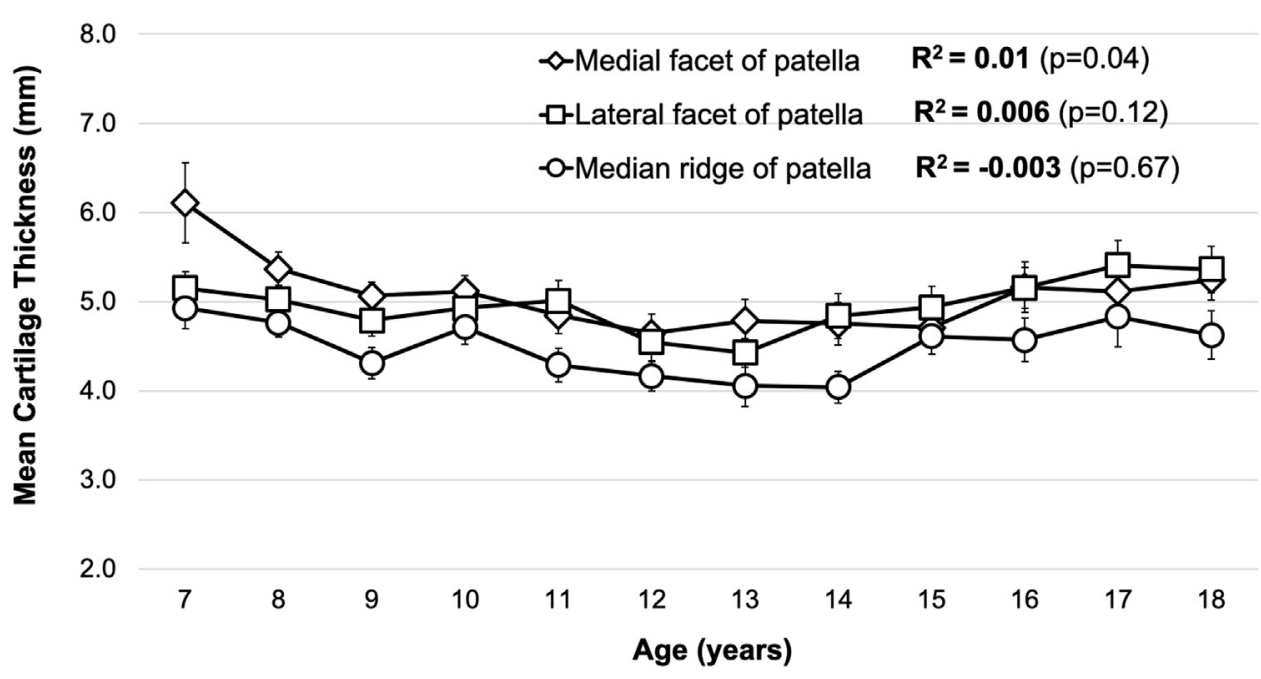

Fig 3. Mean articular cartilage thickness of the patella by age. Mean cartilage thickness at the medial facet, lateral facet, and median ridge are depicted for each year of age. Error bars represent standard error. Adjusted $\mathrm{R}^{2}$ value and $P$ value from linear regression analysis are shown next to the legend for reference. Further details are presented in Table 4.

\section{Discussion}

The results demonstrate that, similar to adults, articular cartilage is thickest at the patella in pediatric and adolescent knees. On the distal femur, articular cartilage is thickest at the lateral trochlea, followed by the medial and lateral femoral condyles. Although patellar cartilage thickness did not correlate with age, distal femoral cartilage thickness significantly decreased with advancing age and skeletal maturity. Articular cartilage in the knee was thicker in male patients compared with female patients at the patella, femur, and tibia.

A few studies have reported anatomic and sex differences in knee articular cartilage that are consistent with these results. In an MRI study of 18 young healthy adult subjects, Faber et al. ${ }^{18}$ showed greatest mean joint knee cartilage thickness at the patella, followed by the trochlea, medial femoral condyle, and lateral femoral condyle. These trends in a small adult population reflect the patterns identified in our current study of pediatric and adolescent knee cartilage thickness but the authors did not perform statistical analysis comparing their anatomic location-specific measurements. With regards to sex differences, cartilage thickness and volume have been shown to be greater in men than in women in the adult population. ${ }^{18-21}$ In a pediatric cohort of 92 children aged 9 to 18 years old, ${ }^{22}$ male patients had $16 \%$ to $31 \%$ greater cartilage volume at the patella and tibia than female patients, after adjusting for age, body mass index, physical activity, and bone area. The reasons for these sex differences in articular cartilage are largely unexplained, but may be due to variations in bone size, physical activity, or hormonal variance.

In a longitudinal study, Jones et al. ${ }^{23}$ evaluated normal knee cartilage development in children. Patellar cartilage volume on sagittal Tl-weighted fat saturation MRI was measured in 74 school children aged 9 to 18 years on 2 occasions at an average interval of 1.6 years. The majority of subjects demonstrated an increase in patellar cartilage volume, with mean volumetric increase of $7.1 \%$ and $6.2 \%$ in male patients and female patients, respectively. Although there was a wide range in rates of cartilage volume increase, younger children, males, and those undertaking more vigorous sports demonstrated greater increases in articular cartilage

Table 4. Linear Regression of Articular Cartilage Thickness by Age

\begin{tabular}{|c|c|c|c|c|c|c|c|c|c|}
\hline & \multicolumn{4}{|c|}{ Linear Regression Model } & \multicolumn{5}{|c|}{ Hierarchical Linear Regression Model With Sex as Added Variable } \\
\hline & Adjusted $\mathrm{R}^{2}$ & B (age) & Y-intercept, mm & $P$ Value & Adjusted $\mathrm{R}^{2}$ & B (Age) & $\mathrm{B}(\operatorname{Sex})$ & Y-intercept, mm & $P$ Value \\
\hline \multicolumn{10}{|c|}{ 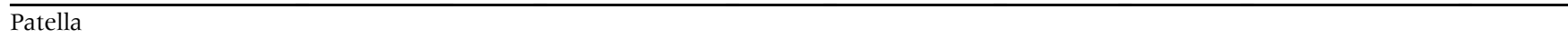 } \\
\hline Lateral facet & 0.006 & 0.03 & 4.6 & .12 & 0.003 & 0.03 & 0.06 & 4.5 & .26 \\
\hline Median ridge & -0.003 & -0.008 & 4.6 & .67 & 0.03 & -0.008 & 0.41 & 4.0 & .006 \\
\hline \multicolumn{10}{|l|}{ Femur } \\
\hline Lateral trochlea & 0.62 & -0.31 & 8.0 & $<.001$ & 0.69 & -0.31 & 0.73 & 6.9 & $<.001$ \\
\hline \multicolumn{10}{|l|}{ Tibia } \\
\hline Lateral tibial plateau & 0.06 & -0.06 & 3.9 & $<.001$ & 0.11 & -0.06 & 0.35 & 3.3 & $<.001$ \\
\hline Medial tibial plateau & 0.27 & -0.12 & 4.4 & $<.001$ & 0.34 & -0.12 & 0.40 & 3.8 & $<.001$ \\
\hline
\end{tabular}

NOTE. $P$ values in bold are statistically significant $(P \leq .05)$. 
Fig 4. Mean articular cartilage thickness of the femur by age. Mean cartilage thickness at the medial femoral condyle, lateral femoral condyle, and lateral trochlea are depicted for each year of age. Error bars represent standard error. Adjusted $\mathrm{R}^{2}$ value and $P$ value from linear regression analysis are shown next to the legend for reference. Further details are presented in Table 4.

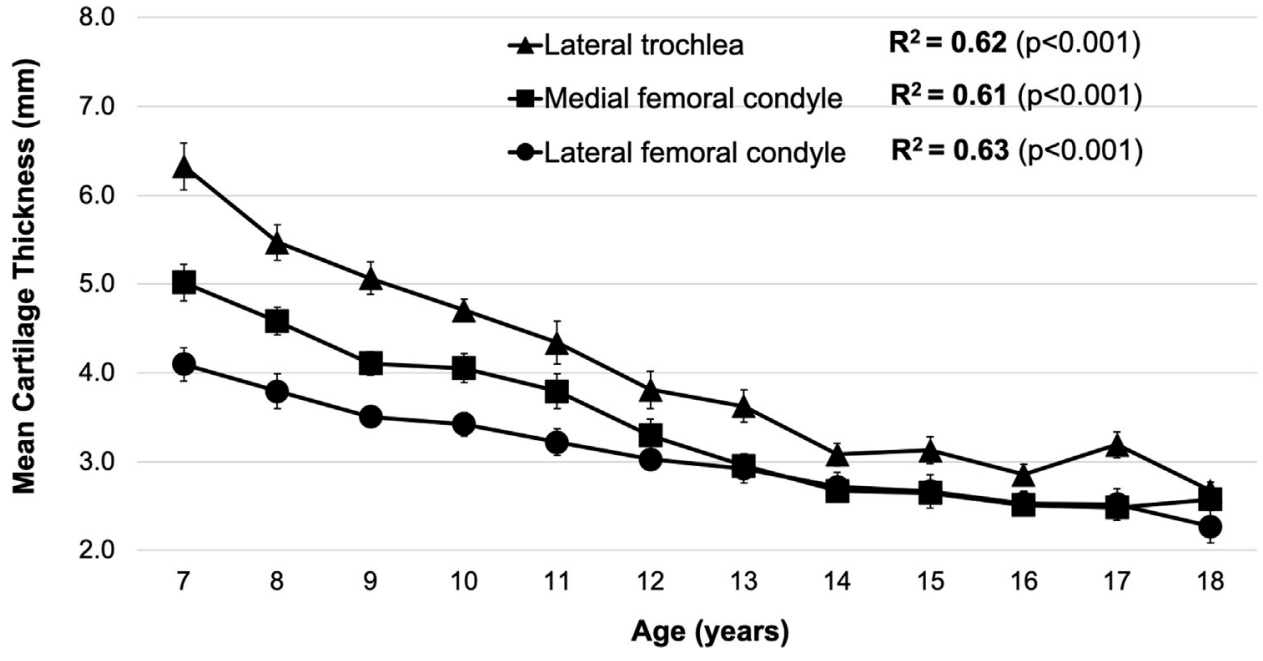

volume. In contrast, the current study showed no significant change in patellar cartilage thickness with age or skeletal immaturity. As the authors themselves state, the observed increase in patellar cartilage volume with age may be due to an increase in subchondral bone area as opposed to an increase in cartilage thickness. ${ }^{23}$ In other words, articular cartilage volume may increase to cover a growing bone surface area in children without any changes in cartilage thickness.

To the contrary, some authors have suggested that in addition to cartilage volume, articular cartilage thickness also may increase with age in adolescents. In a group of 20 elite volleyball players aged 15 to 17 years old with mean 2 years of follow-up, patellar and trochlear cartilage thickness increased annually by $0.8 \%$ in male patients and $0.6 \%$ in female patients. ${ }^{24}$ Similarly, femorotibial cartilage thickness increased annually by $0.8 \%$ in males and $1.4 \%$ in females. ${ }^{25} \mathrm{In}$ comparison, a group of mature athletes aged 40 to 65 years had a decrease in cartilage thickness at the knee at 2-year follow-up, likely reflecting degenerative changes. The authors postulated that articular cartilage in adolescents may be particularly vulnerable to biochemical and biomechanical changes from habitual physical activity, leading to increasing thickness in this group. These results initially appear to contradict our current study, which demonstrates a decrease in cartilage thickness with age. However, differences in the two study designs may in part explain these varying results. The current study is cross-sectional in nature and aims to make conclusions about general trends seen at the population level, as opposed to prospective longitudinal trends at the individual level. Additionally, we included patients aged 7 to 18 years compared with a very exclusive cohort of high-level athletes aged 15 to 17 years. The late adolescents may demonstrate small increases in cartilage thickness differing from the overall trend in the pediatric

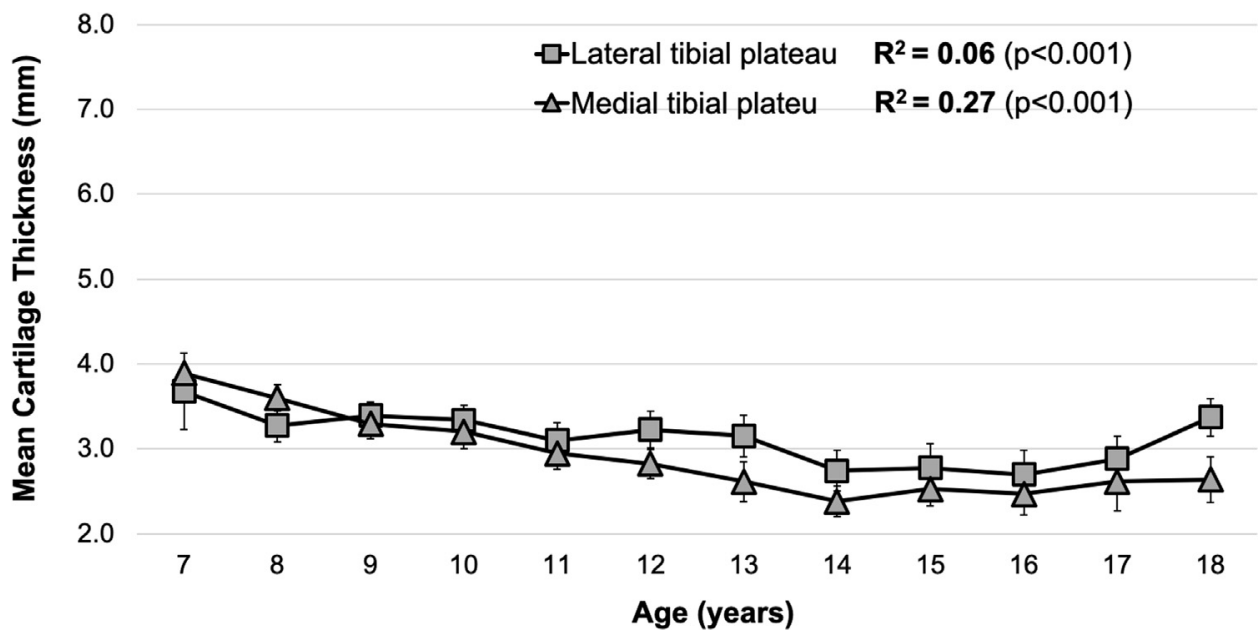

Fig 5. Mean articular cartilage thickness of the tibia by age. Mean cartilage thickness at the medial tibial plateau and lateral tibial plateau are depicted for each year of age. Error bars represent standard error. Adjusted $\mathrm{R}^{2}$ value and $P$ value from linear regression analysis are shown next to the legend for reference. Further details are presented in Table 4 . 
population that were not ascertained or investigated in this study. In fact, although linear regression was used to best model the correlation between age and cartilage thickness at the distal femur and proximal tibia, our graphs demonstrate that the trend starts to plateau by age 14 years.

The aim of this study was to quantify and establish normative values for articular cartilage thickness. Our findings also may be useful in the consideration of matching donor and recipient sites for articular cartilage restoration procedures like OCA. The goal of OCA is to transplant an allograft with viable chondrocytes and underlying subchondral bone into a recipient socket. ${ }^{6}$ Matching a graft to its donor site with respect to size and topography is important to minimizing articular step-off and maintaining near-normal contact pressure. ${ }^{26}$ Studies have shown that an elevation of even $0.5 \mathrm{~mm}$ to $1 \mathrm{~mm}$ over the adjacent articular cartilage at the distal femur can increase contact pressures on the graft up to $50 \%{ }^{27,28}$ In an effort to optimize articular congruity, many surgeons therefore seek hemicondyle grafts matched to the host laterality, mediolateral dimension, and anteroposterior dimension. ${ }^{26,29}$ A few studies have investigated the role of graft subchondral bony thickness in outcomes of OCA. Ackerman et al. ${ }^{30}$ showed that grafts with bony thickness $<5 \mathrm{~mm}$ had 4.9 times greater odds of demonstrating subchondral cystic changes at short-term follow-up. A recent study by Jones et $\mathrm{al}^{31}$ investigated the effect of subchondral bone mismatch at the graft-host interface after OCA transplantation in an adult population with mean age of 30.5 years. The authors noted that there was no significant correlation between maximum graft-host subchondral bone step-off and reoperation rate or graft failure. These observations suggest that differences in cartilage thickness between the graft and surrounding host interface may not adversely affect clinical outcome up to a certain threshold; however, this has not specifically been evaluated in the pediatric population where the cartilage is much thicker in various anatomic regions and can lead to larger magnitudes of mismatch. Typically, OCA plugs are 6 to $8 \mathrm{~mm}$ thick and include 3 to 6 $\mathrm{mm}$ of subchondral bone and 2 to $3 \mathrm{~mm}$ of articular cartilage. ${ }^{30}$ No studies have investigated the impact of graft articular cartilage thickness on outcomes after OCA or OATS.

This current study demonstrates that cartilage thickness at the distal femur significantly decreases with age in pediatric and adolescent knees. In addition to hemicondyle size, cartilage thickness could potentially be an additional variable to consider when matching a graft to its host, particularly in the pediatric population. For OCA, it may be important to match the graft for age and sex in the setting of distal femoral lesions compared with patellar lesions. For patellar lesions, a donor patella of any age may be used rather than using a donor femoral condyle. For OATS, the margin of the lateral trochlea may be an ideal donor site for autograft to the patella given its cartilage thickness and convexity. The patterns of cartilage thickness identified in this study should prompt and inspire further investigation into ideal osteochondral graft location and features in the pediatric and adolescent population that are beyond the scope of this study. As there is limited evidence that a perfectly matched cartilage thickness equates to better functional outcomes, stringent matching criteria should be weighed against practical and logistical concerns, such as time required to find a perfectly matched donor, particular in younger adolescents and children.

The longitudinal reference data presented in this study can aid in preoperative interpretation of knee cartilage under pathologic conditions in pediatric and adolescent patients. An understanding of normal articular cartilage thickness presented in this study can aid sports medicine and pediatric orthopedic surgeons with evaluation of knee MRIs in this population.

\section{Limitations}

This study has several limitations that should be noted. First, this is a retrospective study that evaluated MRI scans from a single North American orthopaedic facility and may not be generalizable to all populations. Demographic and clinical variables such as race/ ethnicity, body mass index, and level of activity may factor into cartilage thickness but were not available for all subjects and therefore not reported. Second, although $16.3 \%$ of knee MRIs were normal, a subset of patients had ACL, meniscal, or medial collateral ligament tears. These knee pathologies may also affect cartilage thickness; in a cohort of ACL injured knees, trochlear cartilage thinned by $1.4 \%$ and patellar cartilage thinned by $0.3 \%$ at 1 year. ${ }^{32}$ However, imaging for ACL tears are often performed close to the time of injury, a period of time in which cartilage thinning related to the ACL injury would not be expected or minimal. Third, there may be limitations associated with using 3-mm MRI slice thickness for measuring articular cartilage thickness; the accurate delineation of cartilage depends on differences in signal relative to adjacent tissue. Fourth, both 3-T and 1.5-T MRI were used in this study. Although some studies have shown a possibility for higher sensitivity and specificity in diagnosis of cartilage lesions with $3-\mathrm{T} \mathrm{MRI},{ }^{33,34}$ it is also more prone to motion artifact which is especially problematic in children. Furthermore, prior studies have demonstrated that MRI using a 1.5-T magnet demonstrates excellent accuracy for diagnosing cartilage pathology. ${ }^{10}$ Finally, as previously mentioned, the cross-sectional nature of this study limits the applicability of its trends in articular cartilage thickness with 
age to individual patients. A prospective longitudinal study would be expensive to execute and limited by a smaller sample size.

\section{Conclusions}

In pediatric and adolescent knees, articular cartilage is thickest at the patella, where it does not strongly correlate with age. In contrast, there is a strong inverse association between increasing age and articular cartilage thickness of the distal femoral condyles. The longitudinal reference data presented in this study can aid in preoperative interpretation of knee cartilage under pathologic conditions in pediatric and adolescent patients.

\section{References}

1. Salzmann GM, Niemeyer P, Hochrein A, Stoddart MJ, Angele P. Articular cartilage repair of the knee in children and adolescents. Orthop J Sport Med 2018;6.

2. Murphy RT, Pennock AT, Bugbee WD. Osteochondral allograft transplantation of the knee in the pediatric and adolescent population. Am J Sports Med 2014;42:635-640.

3. Watson A, Mjaanes JM. Soccer injuries in children and adolescents. Pediatrics 2019:144.

4. Borowski LA, Yard EE, Fields SK, Comstock RD. The epidemiology of US high school basketball injuries, 2005 2007. Am J Sports Med 2008;36:2328-2335.

5. Oeppen RS, Connolly SA, Bencardino JT, Jaramillo D. Acute injury of the articular cartilage and subchondral bone: a common but unrecognized lesion in the immature knee. Am J Roentgenol 2004;182:111-117.

6. Valtanen RS, Arshi A, Kelley BV, Fabricant PD, Jones KJ. Articular cartilage repair of the pediatric and adolescent knee with regard to minimal clinically important difference: A systematic review. Cartilage 2020;11:9-18.

7. Gelber AC, Hochberg MC, Mead LA, Wang NY, Wigley FM, Klag MJ. Joint injury in young adults and risk for subsequent knee and hip osteoarthritis. Ann Intern Med 2000;133:321-328.

8. Mithöfer K, Minas T, Peterson L, Yeon H, Micheli LJ. Functional outcome of knee articular cartilage repair in adolescent athletes. Am J Sports Med 2005;33:1 147-1153.

9. Gudas R, Simonaityte R, Cekanauskas E, Tamosiūnas R. A prospective, randomized clinical study of osteochondral autologous transplantation versus microfracture for the treatment of osteochondritis dissecans in the knee joint in children. J Pediatr Orthop 2009;29:741-748.

10. Potter HG, Linklater JM, Allen AA, Hannafin JA, Haas SB. Magnetic resonance imaging of articular cartilage in the knee. An evaluation with use of fast-spin-echo imaging. J Bone Joint Surg Am 1998;80:1276-1284.

11. Potter HG, Foo LF. Magnetic resonance imaging of articular cartilage: Trauma, degeneration, and repair. Am J Sports Med 2006;34:661-677.

12. Brittberg M, Winalski CS. Evaluation of cartilage injuries and repair. J Bone Joint Surg Am 2003;85:58-69.

13. Argentieri EC, Burge AJ, Potter HG. Magnetic resonance imaging of articular cartilage within the knee. J Knee Surg 2018;31:155-165.
14. Cahill BR, Berg BC. 99m-Technetium phosphate compound joint scintigraphy in the management of juvenile osteochondritis dissecans of the femoral condyles. Am J Sports Med 1983;11:329-335.

15. Pennock AT, Bomar JD, Manning JD. The creation and validation of a knee bone age atlas utilizing MRI. J Bone Joint Surg Am 2018;100:e20.

16. Landis JR, Koch GG. The measurement of observer agreement for categorical data. Biometrics 1977;33:159.

17. Kocher MS, Zurakowski D. Clinical epidemiology and biostatistics: A primer for orthopaedic surgeons. J Bone Joint Surg Am 2004;86-A:607-620.

18. Faber SC, Eckstein F, Lukasz S, et al. Gender differences in knee joint cartilage thickness, volume and articular surface areas: Assessment with quantitative threedimensional MR imaging. Skeletal Radiol 2001;30: 144-150.

19. Cicuttini F, Forbes A, Morris K, Darling S, Bailey M, Stuckey S. Gender differences in knee cartilage volume as measured by magnetic resonance imaging. Osteoarthr Cartil 1999;7:265-271.

20. Cova M, Frezza F, Shariat-Razavi I, Ukmar M, Pozzi Mucelli RS, Dalla Palma L. Magnetic resonance appearance of knee joint hyaline cartilage according to age, sex, and body weight. Radiol Medica 1996;92:171-179.

21. Ding C, Cicuttini F, Scott F, Glisson M, Jones G. Sex differences in knee cartilage volume in adults: role of body and bone size, age and physical activity. Rheumatology (Oxford) 2003;42:1317-1323.

22. Jones G, Glisson M, Hynes K, Cicuttini F. Sex and site differences in cartilage development: A possible explanation for variations in knee osteoarthritis in later life. Arthritis Rheum 2000;43:2543-2549.

23. Jones G, Ding C, Glisson M, Hynes K, Ma D, Cicuttini F. Knee articular cartilage development in children: A longitudinal study of the effect of sex, growth, body composition, and physical activity. Pediatr Res 2003;54: 230-236.

24. Culvenor AG, Wirth W, Maschek S, et al. Longitudinal change in patellofemoral cartilage thickness, cartilage T2 relaxation times, and subchondral bone plate area in adolescent vs mature athletes. Eur J Radiol 2017;92: 24-29.

25. Eckstein F, Boeth H, Diederichs G, et al. Longitudinal change in femorotibial cartilage thickness and subchondral bone plate area in male and female adolescent vs. mature athletes. Ann Anat 2014;196:150-157.

26. Wang D, Coxe FR, Balazs GC, et al. Graft-recipient anteroposterior mismatch does not affect the midterm clinical outcomes of osteochondral allograft transplantation of the femoral condyle. Am J Sports Med 2018;46:2441-2448.

27. Kock NB, Smolders JMH, Van Susante JLC, Buma P, Van Kampen A, Verdonschot N. A cadaveric analysis of contact stress restoration after osteochondral transplantation of a cylindrical cartilage defect. Knee Surg Sport Traumatol Arthrosc 2008;16:461-468.

28. Koh JL, Wirsing K, Lautenschlager E, Zhang L-O. The effect of graft height mismatch on contact pressure following osteochondral grafting: A biomechanical study. Am J Sports Med 2004;32:317-320. 
29. Wang D, Jones KJ, Eliasberg CD, Pais MD, Rodeo SA, Williams RJ. Condyle-Specific matching does not improve midterm clinical outcomes of osteochondral allograft transplantation in the knee. J Bone Joint Surg Am 2017;99A:1614-1620.

30. Ackermann J, Merkely G, Shah N, Gomoll AH. Decreased graft thickness is associated with subchondral cyst formation after osteochondral allograft transplantation in the knee. Am J Sports Med 2019;47:2123-2129.

31. Jones KJ, Coxe F, Wang D, Williams R. Osteochondral allograft-host interface mismatch does not affect reoperation rates when using less restrictive graft matching criteria. Presented at the 15th Annual World Congress International Cartilage Regeneration and Joint
Preservation Society, Vancouver, British Columbia, October 2019.

32. Frobell RB, Le Graverand M-P, Buck R, et al. The acutely ACL injured knee assessed by MRI: changes in joint fluid, bone marrow lesions, and cartilage during the first year. Osteoarthr Cartil 2009;17:161-167.

33. Wong S, Steinbach L, Zhao J, Stehling C, Ma CB, Link TM. Comparative study of imaging at $3.0 \mathrm{~T}$ versus $1.5 \mathrm{~T}$ of the knee. Skeletal Radiol 2009;38:761-769.

34. Cheng Q, Zhao FC. Comparison of 1.5- and 3.0-T magnetic resonance imaging for evaluating lesions of the knee: A systematic review and meta-analysis (PRISMA-compliant article). Medicine (Baltimore) 2018;97:e12401. 